\title{
Probing the driving region in hot subdwarf stars through nonadiabatic asteroseismology: the principle of the method
}

\author{
S. Charpinet ${ }^{1}$, G. Fontaine ${ }^{2}$, and P. Brassard ${ }^{2}$
}

\author{
${ }^{1}$ Laboratoire d'Astrophysique de Toulouse-Tarbes, Université de Toulouse, CNRS, 14 Av. E. Belin, 31400 Toulouse, France \\ e-mail: stephane.charpinet@ast.obs-mip.fr \\ 2 Département de Physique, Université de Montréal, C.P. 6128, Succ. Centre-Ville, Montréal QC H3C 3J7, Canada \\ e-mail: [fontaine; brassard]@astro.umontreal.ca
}

Received 2 June 2008 / Accepted 26 October 2008

\begin{abstract}
Context. The $\kappa$-mechanism that drives pulsations in hot subdwarf stars is closely linked to the action of diffusive processes, including radiative levitation, which modulate the local contents of heavy elements in the stellar envelope. Iron, in particular, is important for its dominant contribution to the Z-bump feature in the envelope opacity, although other iron-peak elements, such as nickel for instance, may also play a significant role.

Aims. Our main goal is to evaluate the potential of nonadiabatic asteroseismology for studying diffusive phenomena in these stars. In this exploratory work, we consider iron as a test case to establish the principle of the method.

Methods. From model experiments, we explore the behavior of the pulsation engine under assumed local iron enrichments in the $Z$-bump layers, and we show how this may be related, through the period range of unstable modes, to some observed properties, i.e., the range of periods effectively detected, characterizing hot pulsating subdwarf stars. This connects nonadiabatic physics with observables.

Results. We demonstrate that the strength of the pulsation engine is predominently controled by the amount of heavy metals (iron in our experiments) present at the Z-bump location, the chemical composition in other parts of the star being irrelevant to the process. We also show that this property can be used to probe directly the amount of metals present in this particular region, irrespective of the physical process involved to produce such abundances. In particular, we show that limits on the abundances needed for the onset of pulsations can be estimated. These can even be derived for individual stars based on their observed pulsation period range, as illustrated with two well-studied pulsating sdB stars: PG 1325+101 and Feige 48.

Conclusions. We conclude by emphasizing the strong potential of nonadiabatic asteroseismology for hot subdwarf stars, which may hold the key for better understanding diffusive and competing mixing processes in stellar envelopes.
\end{abstract}

Key words. stars: oscillations - stars: subdwarfs

\section{Introduction}

Among oscillating stars, hot pulsating subdwarfs are singular in that radiative levitation of heavy elements (especially iron) is expected to play a central role explaining the existence of the pulsations themselves. The intimate relationship between the envelope metal content and the pulsation driving process was first established by Charpinet et al. (1996) for the short-period B subdwarf pulsators (the V361 Hya or EC14026 stars; Kilkenny et al. 1997). It was demonstrated that $p$-mode instabilities can occur in sdB stellar models through the action of a classical $\kappa$-mechanism involving mainly partial ionization of the M-shell electrons of iron, the latter resulting in a sharp peak, often called the Z-bump, in the Rosseland opacity profile. It was also shown that a solar content of metals in the envelope is unable, however, to produce enough driving to globally destabilize modes, thus implying that an additional process must be involved to boost the $\kappa$-effect. This process is most likely radiative levitation, which is expected to act efficiently in the stable radiative envelopes of hot subdwarf stars. In competition with other processes, such as gravitational settling and weak stellar winds, radiative levitation is believed to be responsible for the well known atmospheric abundance anomalies observed in sdB stars (see, e.g., Blanchette et al. 2008, and references therein). It is also expected to produce reservoirs of levitating elements below the photosphere, although these reservoirs cannot be observed directly as these optically thick layers remain hidden to spectroscopic studies. In particular, iron tends to accumulate nonuniformely at relatively high extrasolar abundances in the stellar envelope with the effect of powering up the driving mechanism and producing efficient excitation for pulsation modes (Charpinet et al. 1997). Remarkably, the same $\kappa$-effect involving radiative levitation is likely responsible for the driving of $g$-modes in the long period sdB pulsators (Fontaine et al. 2003) discovered by Green et al. (2003), and for the driving of very short period $p$-modes in the newly discovered class of hot pulsating sdO stars (Fontaine et al. 2008), whose only known member thus far is the star SDSS J160043.6+074802.9 (Woudt et al. 2006).

The identification of the main constituents producing the driving of pulsations in hot $\mathrm{sdB}$ stars inspired the development of new stellar models dealing with the important role of radiative levitation (the so-called "second generation models" introduced by Charpinet et al. 1997). These models are constructed around the assumption that a state of diffusive equilibrium is reached between radiative levitation and gravitational settling (thus disregarding, at this stage, other potentially competing processes), leading to stable nonuniform abundance profiles for chemical species in the stellar envelope. It is indeed expected that this state 
is reached over timescales much shorter than the typical lifetime of sdB stars $\left(\sim 10^{8} \mathrm{yr}\right)$, as shown by Fontaine et al. (2006a) from time-dependent diffusion calculations. Another simplification introduced in the second generation models is that only iron is allowed to levitate in a pure hydrogen envelope, a choice justified by the facts that iron is the dominant contributor to the $Z$-bump opacity, and that helium is well known to be very underabundant in the envelopes/atmospheres of sdB stars (a situation presumably caused by rapid sedimentation). The real picture might be more complicated, however, as pointed out recently by Jeffery \& Saio (2006a,b, 2007) who argued that nickel, despite being some $\sim 20$ times less abundant than iron, may also contribute a significant fraction of the stellar gas opacity in the $Z$-bump region. They also noticed important differences between the OPAL opacities (Iglesias \& Rogers 1996) and the most recent OP opacities (Badnell et al. 2005) having a direct impact on the properties of the pulsation driving mechanism. In particular, they found indications that models using the OP opacities may better reproduce the position of the instability region for the long period g-mode sdB pulsators, although they did not include radiative levitation in their calculations.

Despite the potential shortcomings and uncertainties alluded to above, second generation models have constituted a substantial improvement over canonical stellar structures based on chemically uniform abundances for the study of $\mathrm{sdB}$ pulsators. For instance, the class properties of pulsating sdB stars are globally well reproduced with these structures (see, e.g., Charpinet et al. 2001, 2007), and they have been quite successfully used for detailed asteroseismic studies of several EC14026 stars, as examplified by the most recent analysis of the star PG 1336-018 (Charpinet et al. 2008). Yet, model improvements are still necessary to tackle some of the pending issues in the field. Among these, understanding why only a minority of stars that fall within the empirical EC14026 instability domain in the $\log g-T_{\text {eff }}$ plane actually pulsate is one of the current challenges (although a solution might reside in the presence of weak stellar winds in that case; Fontaine et al. 2006b). Another issue concerns the actual extent of the observed instability strip for the EC14026 stars, which is currently narrower than the predicted one, and which can be correlated with the fact that the observed period ranges in individual stars are also usually narrower than predicted. All of this is plausibly linked to approximations and factors that control and modulate the efficiency of the driving mechanism, themselves resulting from the interplay of various competing processes not necessarily included in the current second generation models.

In this context, the goal of the present paper is to emphasize a remarkable property of the driving mechanism at work in pulsating hot subdwarf stars. This property, which has been overlooked in the past, can potentially provide indications as to how to progress on some of the issues mentioned above. For that purpose, we reinvestigate, in Sect. 2, the driving mechanism in hot B subdwarf stars in order to clearly identify the dominant factor that controls the efficiency of the driving mechanism. We then demonstrate, in Sect. 3, that the specific properties of the driving mechanism at work in these stars offer a unique opportunity to directly probe the chemical content in the subphotospheric (and therefore hidden) layers where the $Z$-bump occurs. We illustrate the principle of the method in the framework of our second generation models, while pointing out, however, that this probing is currently prone to systematic effects arising from still unsettled uncertainties that persist at the level of the microphysics itself (induced mainly by both the OP versus OPAL problem and the relative contribution of $\mathrm{Ni}$ to the $Z$-bump opacity mentioned previously). We finally discuss important implications of this finding in Sect. 4.

\section{The main factor controling the pulsation engine}

Our investigation proceeds within the framework of our second generation models. Consequently, we assume at this stage that iron is the only significant contributor to the Z-bump, and therefore to the driving mechanism. Moreover, we base our discussion on models employing the OPAL opacities. These assumptions are sufficient for the present purpose, which is to demonstrate the validity of the proposed approach. The possible influences of other chemical species (notably nickel) and of using OP instead of OPAL opacities will be briefly discussed in the next section and investigated quantitatively in detail in a subsequent paper.

Given that the driving mechanism at work in hot pulsating subdwarfs relies on radiative levitation producing local iron enrichments in the stellar envelope, a natural question is: what factors effectively control the strength of the pulsation engine? Intuitively, one would think that the amount of iron levitating in the Z-bump region, where the driving/damping takes place, plays the major - if not exclusive - role. Experimenting with this idea, we considered a representative sdB star with parameters $T_{\text {eff }}=34000 \mathrm{~K}, \log g=5.8, M=0.48 M_{\odot}$, and $\log q(H) \simeq$ $\log \left(M_{\text {env }} / M\right)=-4.0$, typical for an object located near the center of the EC14026 instability domain (e.g., Charpinet et al. 2001). A second generation model incorporating iron profiles from diffusive equilibrium (hereafter the "reference model") was calculated with the above parameters. The iron profile and corresponding Rosseland opacity profile for this model are shown as blue solid curves in Fig. 1. In parallel, we also computed a series of structures (hereafter called "Z-bump enhanced models") with the same set of parameters. The latter models, however, were built to explore the effect of increasing the amount of iron only in the Z-bump region, while keeping it solar everywhere else in the envelope. For that purpose, the iron abundance distribution in the envelope was artificially set to a step-like profile centered at the tip of the iron opacity peak $(\log q \sim-10$ in the present case), extending down to $\log q=-9$ and up to $\log q=-11$, and connecting with exponential tails to the uniform solar value in order to fully cover the region occupied by the Z-bump (see Fig. 1). The height of the step profile is defined by a factor, $f$, that is applied to the reference iron content, the latter being the solar value $\log \mathrm{Fe} / \mathrm{H}=-4.5$. The tails in these artificial abundance distributions are only added to avoid discontinuities (and their associated numerical complications) in physical quantities such as the mean Rosseland opacity run. Their arbitrary shape and extent have no influence on what follows. Moreover, the uniform abundance distribution assumed in the $Z$-bump region is meant to reflect an "average" content of iron that is present in those specific layers. No sophisticated modeling of a nonuniform distribution in this relativery narrow region is attempted at this stage, especially as simple tests indicate that such refinements have essentially no impact on the results presented below, unless very steep distributions are considered. In the present context (see below), the average content of iron is the important quantity to consider. Obviously, such models are unphysical and should not be used for precise asteroseismic purposes. They turn out to be particularly interesting, however, for pinning down the main contributing factor controling the efficiency of the pulsation engine and for their potential use in the context of nonadiabatic asteroseismology (see below). Nonadiabatic properties for representative $\ell=0, k=0-9 p$-modes were computed for all these models (see Fig. 1 caption for details), some of which are summarized 
Table 1. Nonadiabatic properties for our reference model, its corresponding $Z$-bump enhanced model (Model $\times 12.11$ ), and two other models where the iron enrichment factor in the $Z$-bump region is $\times 1.0$ (i.e., no enrichment) and $\times 100$, respectively.

\begin{tabular}{|c|c|c|c|c|c|c|c|c|c|}
\hline \multirow[b]{2}{*}{$\ell$} & \multirow[b]{2}{*}{$k$} & \multicolumn{2}{|c|}{$\overline{\text { Reference model }}$} & \multicolumn{2}{|c|}{ Model $\times 12.11$} & \multicolumn{2}{|c|}{$\overline{\text { Model } \times 1.0}$} & \multicolumn{2}{|c|}{$\overline{\text { Model } \times 100}$} \\
\hline & & $P(\mathrm{~s})$ & $\sigma_{I}(\mathrm{rad} / \mathrm{s})$ & $P(\mathrm{~s})$ & $\sigma_{I}(\mathrm{rad} / \mathrm{s})$ & $P(\mathrm{~s})$ & $\sigma_{I}(\mathrm{rad} / \mathrm{s})$ & $P(\mathrm{~s})$ & $\sigma_{I}(\mathrm{rad} / \mathrm{s})$ \\
\hline 0 & 9 & 58.56 & $+7.398 \times 10^{-4}$ & 58.60 & $+7.257 \times 10^{-4}$ & 58.10 & $+1.291 \times 10^{-3}$ & 59.15 & $+1.662 \times 10^{-4}$ \\
\hline 0 & 8 & 64.65 & $+3.237 \times 10^{-4}$ & 63.88 & $+2.803 \times 10^{-4}$ & 63.38 & $+1.022 \times 10^{-3}$ & 64.24 & $-7.767 \times 10^{-5}$ \\
\hline 0 & 7 & 70.33 & $+8.226 \times 10^{-5}$ & 68.88 & $+1.307 \times 10^{-4}$ & 68.07 & $+6.105 \times 10^{-4}$ & 69.85 & $-2.964 \times 10^{-4}$ \\
\hline 0 & 6 & 77.46 & $-9.966 \times 10^{-6}$ & 77.47 & $-1.881 \times 10^{-5}$ & 75.41 & $+4.658 \times 10^{-4}$ & 78.96 & $-3.974 \times 10^{-4}$ \\
\hline 0 & 5 & 88.79 & $-6.080 \times 10^{-5}$ & 87.57 & $-5.275 \times 10^{-5}$ & 86.07 & $+1.465 \times 10^{-4}$ & 88.21 & $-1.995 \times 10^{-4}$ \\
\hline 0 & 4 & 98.29 & $-3.430 \times 10^{-5}$ & 95.30 & $-5.397 \times 10^{-5}$ & 94.47 & $+4.676 \times 10^{-5}$ & 96.97 & $-2.738 \times 10^{-4}$ \\
\hline 0 & 3 & 110.53 & $-3.100 \times 10^{-5}$ & 109.57 & $-3.659 \times 10^{-5}$ & 108.89 & $+2.423 \times 10^{-5}$ & 111.56 & $-1.921 \times 10^{-4}$ \\
\hline 0 & 2 & 134.76 & $-5.258 \times 10^{-6}$ & 129.59 & $-3.205 \times 10^{-6}$ & 131.11 & $+1.965 \times 10^{-6}$ & 128.03 & $-1.546 \times 10^{-5}$ \\
\hline 0 & 1 & 145.75 & $-1.131 \times 10^{-6}$ & 139.24 & $-1.594 \times 10^{-6}$ & 141.06 & $+1.011 \times 10^{-6}$ & 137.25 & $-6.687 \times 10^{-6}$ \\
\hline 0 & 0 & 174.36 & $-9.657 \times 10^{-8}$ & 191.78 & $-2.138 \times 10^{-8}$ & 194.69 & $+4.768 \times 10^{-8}$ & 188.48 & $-5.859 \times 10^{-8}$ \\
\hline
\end{tabular}

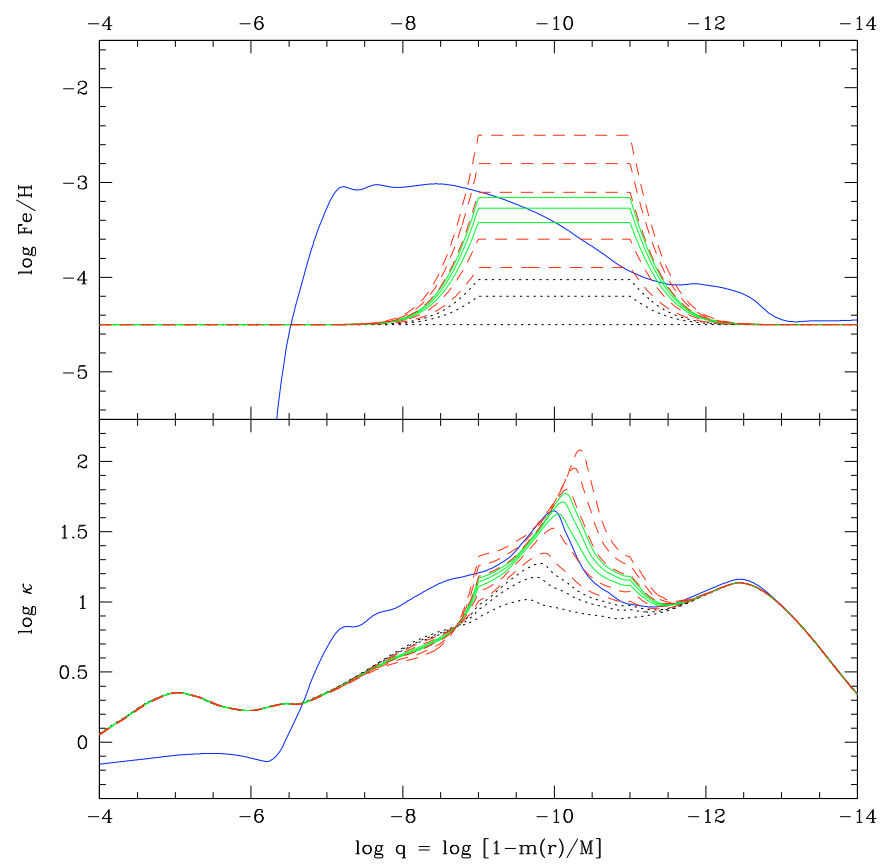

Fig. 1. Upper panel: iron content, $\log \mathrm{Fe} / \mathrm{H}$ (in number), as a function of fractional mass depth, $\log q$, for a series of models. The blue solid curve shows the iron profile predicted from equilibrium between gravitational settling and radiative levitation (the reference model). Other profiles are from models constructed to produce an enrichment of iron only in the Zbump region (and solar everywhere else). From bottom to top, the black dotted-lined step profiles represent models with local enrichments of $\times 1, \times 2, \times 3$ the solar value $(\log \mathrm{Fe} / \mathrm{H}=-4.5)$ where no mode driving is found. The red dashed-lined step profiles are models with Fe local enrichments of $\times 4, \times 8, \times 25, \times 50, \times 100$ the solar value showing $p$-modes instabilities. The green solid-line step profiles are models with local Fe enrichments of $\times 12, \times 17$, and $\times 22$ showing a range of excited $p$-modes similar to the reference model. Lower panel: logarithm of the Rosseland opacity, $\log \kappa$, as a function of fractional mass depth for the same series of models. Color and curve conventions are the same as above.

in Table 1. We recall that in the context of nonadiabatic pulsation theory, the imaginary part of the complex eigenfrequency, $\sigma_{I}$, carries information on the global stability of a mode (while the real part gives the period, $P=2 \pi / \sigma_{R}$ ). If the sign of $\sigma_{I}$ is negative (positive), it implies that the mode of interest is excited (damped).

In this exploration, calculations show that mode instabilities do not occur in the $Z$-bump enhanced models with $f$-factors of $\times 1$ (i.e., no iron overabundance in the $Z$-bump region), $\times 2$, and

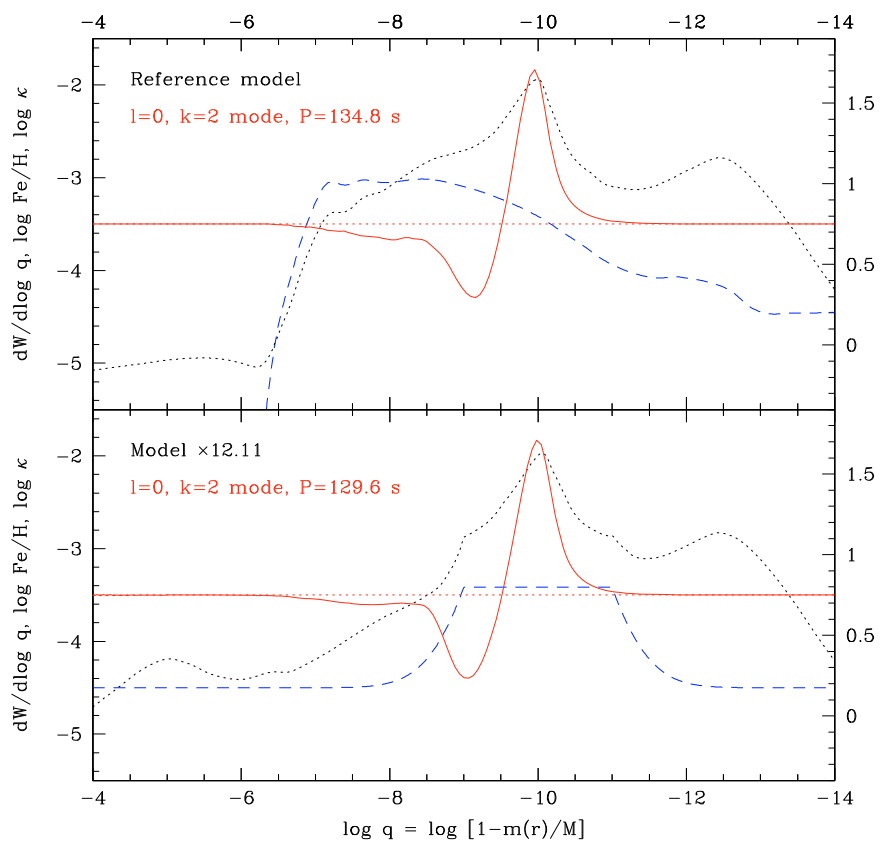

Fig. 2. Driving and damping structure for a representative $\ell=0, k=2$ globally excited $p$-mode (see Table 1 ) from the reference model (upper panel) and from its corresponding Z-bump enhanced model $(\times 12.11$ the solar value, lower panel). The red solid curve shows the integrand of the work integral of the mode as a function of fractional mass depth. The blue dashed curve is the iron abundance profile, to be read on the LHS axis. The black dotted curve gives the run of the Rosseland opacity, to be read on the RHS axis.

$\times 3$ (black dotted profiles in Fig. 1). Instabilities appear for local iron enrichments of $\times 4$ and beyond. We also find that the Z-bump enhanced models with $\times 12, \times 17$, and $\times 22$ iron enrichments (green solid curves in Fig. 1) are those which excite the same band of modes as the reference model. Models with lower (higher) $f$-factors are found to excite a narrower (wider) band. The important fact, and this clearly appears in the upper panel of Fig. 1, is that the amounts of iron enrichment in the $\times 12$, $\times 17$, and $\times 22$ models correspond approximately to the average content of iron that is levitating in the $Z$-bump region according to diffusive equilibrium calculations included in the reference model. A closer look at the reference model indicates that the quantity of iron levitating near $\log q \sim-10$ (i.e., at the top of the opacity bump) is indeed $\sim 12.11 \times$ the solar value. Later, we will examine a $Z$-bump enhanced model with this particular iron enrichment factor (Model $\times 12.11$; see Table 1, Fig. 2, and below). 
We finally note that the shape of the Z-bump in the corresponding opacity profiles (see lower panel of Fig. 1) is very similar to the profile observed in the reference model. This is especially true for the $Z$-bump enhanced model with an iron enrichment of $\times 12$.

The detailed nonadiabatic properties of Model $\times 12.11$ are given in Table 1. Despite noticeable differences in the periods (mostly for the $k \sim 0-4$ modes) that, not surprisingly, disqualify all the $Z$-bump enhanced models for standard asteroseismic purposes based on precise period matching, this structure and the reference model show strong similarities in terms of mode excitation, both having driven $p$-modes with radial orders $k=0-6$ $(P \sim 192-77 \mathrm{~s})$. We show in Fig. 2 the driving/damping structure for the representative $\ell=0, k=2$ mode in the reference model (upper panel) and in Model $\times 12.11$ (lower panel). This plot again illustrates the similar shapes of the Z-bump structures in the opacity profiles for these two models. In addition, it demonstrates, through the similar shapes of the integrand of the work integral associated with this mode (the red solid curves), that the mode driving structure is also essentially unchanged. We recall that this quantity measures the energy locally gained (lost) in each stellar layer by the mode over a pulsation cycle. Positive values indicate local driving (here associated with the $Z$-bump structure) and negative values show the damping regions (here below the $Z$-bump).

From these experiments, we draw an important conclusion: the strength of the pulsation engine at work in hot subdwarf stars is essentially controled by a single factor largely dominated by the amount of opaque metals (iron and eventual additional contributors such as nickel) present in the Z-bump region only. The chemical composition in other stellar layers is irrelevant as far as mode excitation is concerned, although it plays an essential role for precise determinations of the pulsation periods. Thus, the nonadiabatic properties of pulsating hot subdwarfs (including not only the rapid sdB pulsators considered here, but also the long period $\mathrm{sdB}$ and the new $\mathrm{sdO}$ pulsators) only depend on the average metal content present in the very localized subphotospheric layers where the $Z$-bump occurs, regardless of the physical process that ultimately leads to such local abundances.

\section{Probing the metal content in the Z-bump region}

The above conclusions pave the way for the application of a new nonadiabatic asteroseismic approach to the study of hot subdwarf stars. Figure 3 illustrates the basics of this approach for the representative sdB star model explored in the previous section. On the left hand side of this plot, the period spectrum for the reference model (cf., Table 1) is represented vertically, with the driven modes shown as blue filled circles. The other sequences of period spectra are from Z-bump enhanced models with, from left to right, increasing values for the iron enrichment factor (see caption of Fig. 3 for details).

This figure allows us to identify two interesting events in terms of mode stability. The first one, occuring between enrichments factors of $\times 3$ and $\times 3.1$, is the onset of mode instabilities. Below an enrichment factor of $\times 3.1$, no pulsations are expected in these stars. This sets a limit of the average amount of levitating iron that must be present in the $Z$-bump region for both pulsating (above) and nonpulsating (below) sdB stars with parameters $T_{\text {eff }} \sim 34000 \mathrm{~K}$ and $\log g \sim 5$.8. The second event is reached when further increasing the enrichment factor. The domain of unstable periods then naturally expands, mainly to modes with higher radial orders, until it reaches a point where the band of driven modes corresponds to the one seen in the reference model.

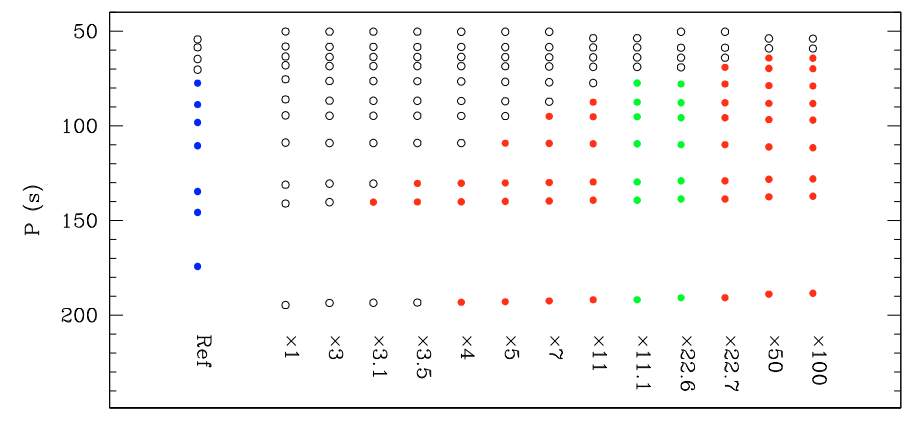

Fig. 3. Period spectra and mode stability. Our reference model is shown on the LHS of this plot. Other models are Z-bump enhanced structures with iron enrichment factors of, from left to right, $\times 1, \times 3, \times 3.1, \times 3.5$, $\times 4, \times 5, \times 7, \times 11, \times 11.1, \times 22.6, \times 22.7, \times 50$, and $\times 100$, respectively. Black open circles indicate damped modes and colored filled circles correspond to driven modes. Blue is used for the reference model, red for models that do not match the range of excited modes in the reference model, and green for models that match this range.
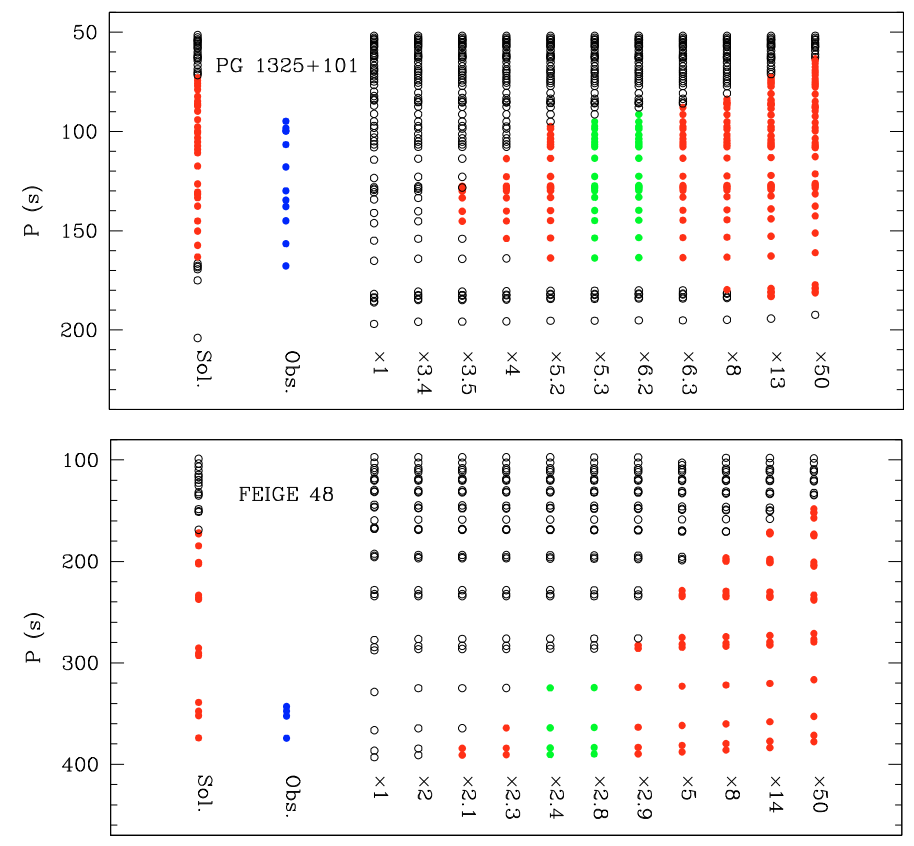

Fig. 4. Nonadiabatic asteroseismology of the short-period sdB pulsators PG 1325+101 (upper panel) and Feige 48 (lower panel). Blue filled circles indicate the observed periods, the model on the LHS is the optimal solution derived from asteroseismology, and the other models are Z-bump enhanced structures with enrichment factors given in the respective panels. Series with green filled circles indicate models that match the range of observed modes in the considered star.

As shown in Fig. 3 (models with green filled circles), this happens in a limited range - between $\times 11$ and $\times 22.7$ - for the enrichment factor. We recall that a factor $\times 12.11$ of iron is indeed levitating at the tip of the opacity bump in the reference model, where the driving effect is maximum.

The same argumentation can be applied to real pulsators assuming that the sensitivity of the observations has been sufficient to determine the whole range of excited modes in these stars. We illustrate this in Fig. 4 for two well-studied short period sdB stars: PG $1325+101$ at $T_{\text {eff }} \simeq 35050 \mathrm{~K}, \log g \simeq 5.811$ (upper panel; Charpinet et al. 2006) and Feige 48 at $T_{\text {eff }} \simeq 29580 \mathrm{~K}$, $\log g \simeq 5.462$ (lower panel; Van Grootel et al. 2008). The observed period spectrum for each star is given as blue filled circles. It is compared with the period spectrum of their respective 
best model solutions proposed in the publications cited above. In both cases, the range of excited periods in that model is clearly more extended than the observed range, a typical situation with the second generation models. By producing two series of $Z$-bump enhanced models, each adapted to the star under consideration, we can determine the average iron content needed in the $Z$-bump region, first to provoke the onset of pulsation mode instabilities (this occurs at factors of $\times 3.5$ and $\times 2.1$ for PG 1325+101 and Feige 48, respectively, thus showing that this limit varies as a function of $\log g$ and $T_{\text {eff }}$ ) and, second, to match the observed period range. The latter occurs in the ranges $\times 5.2-$ 6.3 for PG $1325+101$ and $\times 2.3-2.9$ for Feige 48 . Note that for the latter estimations, another assumption must be made which is that all modes predicted unstable by the nonadiabatic linear pulsation theory can indeed be excited to observable amplitudes in the real star. The validity of this assumption, or possible corrections to it, rely, strictly speaking, on a detailed nonlinear description of stellar oscillations that can also predict the amplitudes of the excited modes (the linear approach cannot). This nonlinear theory is not available at the present stage, however.

Hence, through the new nonadiabatic asteroseismic approach that we propose in this paper, we obtain independent estimates of the amount of iron present at the location of the Z-bump, regardless of the physical process involved to create this particular abundance. For PG $1325+101$ we estimate an iron enrichment of $\times 5.75 \pm 0.55$ over the solar value, i.e., $\log \mathrm{Fe} / \mathrm{H}=-3.74 \pm 0.04$. For Feige 48, we find an enrichment of $\times 2.60 \pm 0.30$ over the solar value, i.e., $\log \mathrm{Fe} / \mathrm{H}=-4.09 \pm 0.05$. Note that because the $Z$-bump region responsible for the mode driving has a finite width (although relatively narrow in terms of radius and mass fraction), the obtained iron abundance represents an average estimate in the range effectively covered by the Z-bump. Therefore, such a measurement should be representative of the local iron abundance distribution as long as the latter remains relatively flat within this particular region.

The values given above illustrate the potential of the method. At this stage, they should not be taken at face value, as they are likely subject to systematic effects that need to be investigated in detail. These effects are mainly related to the facts that 1) differences between OPAL and OP opacities may be sufficiently large to significantly affect the derived values, and 2) the contribution of other species, in particular nickel, to the opacity bump may be nonnegligible. The uncertainty associated with the opacities should disappear after the issue concerning which, of the OPAL or OP data set, is the most realistic one is settled. At this stage, we simply note that using OP opacities instead of the OPAL data, as we did here, may lead to a decrease of the derived values for the iron abundance because the driving mechanism appears more efficient for a given amount of iron in the Z-bump region when OP data are used (see Jeffery \& Saio 2007). It is impossible at this stage to say if this change will be significant or not. We also stress that if nickel has a significant contribution to the Z-bump opacity and to the driving mechanism, the basic principle of the proposed method still applies. The measured quantity, however, would then correspond to the mixture of $\mathrm{Fe}+\mathrm{Ni}$ that would be present in the driving region (instead of only iron as given in our examples above). This would mean, for given opacities and provided that the $\mathrm{Ni} / \mathrm{Fe}$ ratio can be estimated from other considerations, that both the amount of nickel and of iron present in that region could potentially be estimated.

Addressing the abovementioned issues will require a vast exploration of these effects using improved sets of models. This would go far beyond the scope of the present paper, but because of the interesting potential of this nonadiabatic asteroseismic approach, we plan additional investigations in that direction.

\section{Conclusion and prospects}

In this paper, we demonstrated that the pulsation engine at work in oscillating $\mathrm{sdB}$ and $\mathrm{sdO}$ stars is predominently controled by the average amount of metal (mostly iron) present in the subphotospheric layers where the Z-bump occurs, irrespective of the precise mechanism responsible for building up such an abundance. The chemical composition in other parts of the star is essentially irrelevant to the process. We stress that this remarkable property, which has been overlooked thus far, is of great interest because it opens up a new independent way of probing the interior of hot subdwarf stars, as demonstrated in this paper. This is nonadiabatic asteroseismology in action.

Indeed, through the material presented in this paper, we have established the principle of this nonadiabatic approach. In particular, we showed that direct and independent probing of the chemical content in that normally hidden subphotospheric $Z$-bump region is feasible using an observable property of pulsating subdwarf stars (the observed period range of the oscillations). This capacity should be of great help in better understanding the interplay of various diffusive and mixing processes that compete in the envelope of such stars. Currently, we can only observe the effects of such competing processes in the atmospheric layers. The nonadiabatic method proposed here should therefore constitute a very useful complementary approach to the study of abundance anomalies in hot subdwarf stars. In particular, we, again, point out the current discrepencies that exist between the second generation models and the observed nonadiabatic properties of pulsating sdB stars, the former predicting wider bands of unstable modes as well as larger instability strips than observed. Following the ideas developed here, it should become obvious that these discrepancies are likely due to the presence of too much iron in the $Z$-bump region in these models. This is due, most likely, to the simplifying hypotheses adopted for the treatment of diffusion in these stars. A better treatment, with the inclusion of other potentially competing process, such as the levitation of other elements or additional mixing mechanisms slowing down or limiting the formation of reservoirs in equilibrium, are likely to decrease the amount of iron (and of other opaque metals) effectively accumulating in the $Z$-bump region. Hence, the local information provided by the nonadiabatic approach, which is independent of the physical mechanisms responsible for the abundance stratification as we showed in this paper, should become important in improving the models at that level. Model improvements are also needed to achieve a better accuracy in fitting the pulsation periods observed in EC14026 stars (see, e.g., the detailed asteroseismic study proposed by Charpinet et al. 2008). Typically, there is still approximately an order of magnitude between the best achievable match to the observed periods and the accuracy at which these periods are effectively measured. We strongly suggest here that both issues must be linked, as the pulsation periods are sensitive to the global distribution of the opaque chemical species in the stellar envelope (and not only by the amount of opaque metal present in the Z-bump region, as it is the case for the nonadiabatic properties). Hence, the uncertainties associated with the modeling of diffusive and mixing processes in sdB envelopes can be investigated from these two complementary approaches. More generally, this may help shed new light on the effects of various mixing processes that may be at work in stars in general (see, e.g., Dupret 2008, for a review of current challenges in modern stellar physics). In this context, 
pulsating hot subdwarfs may be useful in resolving these problems.

Further studies to fully unveil the potential of nonadiabatic asteroseismology for hot subdwarf stars are thus highly desirable. In particular, we plan in a future investigation to calibrate this new approach by evaluating the impact of systematic effects linked to current uncertainties at the level of the microphysics of the models (the OP versus OPAL opacities issue) and to the possible contribution of other chemical species, such as nickel, to the driving mechanism.

Acknowledgements. This work was supported in part by the NSERC of Canada. G.F. also acknowledges the contribution of the Canada Research Chair Program.

\section{References}

Badnell, N. R., Bautista, M. A., Butler, K., et al. 2005, MNRAS, 360, 458 Blanchette, J.-P., Chayer, P., Wesemael, F., et al. 2008, ApJ, 678, 1329

Charpinet, S., Fontaine, G., Brassard, P., \& Dorman, B. 1996, ApJ, 471, L103
Charpinet, S., Fontaine, G., Brassard, P., et al. 1997, ApJ, 483, L123 Charpinet, S., Fontaine, G., \& Brassard, P. 2001, PASP, 113, 775

Charpinet, S., Silvotti, R., Bonanno, A., et al. 2006, A\&A, 459, 565

Charpinet, S., Fontaine, G., Brassard, P., et al. 2007, Commun. Asteroseismol., 150,241

Charpinet, S., Van Grootel, V., Reese, D., et al. 2008, A\&A, 489, 377

Dupret, M.-A. 2008, 5th HELAS Workshop in Wroclaw, Poland, in press Fontaine, G., Brassard, P., Charpinet, S., et al. 2003, ApJ, 597, 518

Fontaine, G., Brassard, P., Charpinet, S., \& Chayer, P. 2006a, Mem. Soc. Astron. It., 77, 49

Fontaine, G., Green, E. M., Chayer, P., et al. 2006b, Balt. Astron., 15, 211

Fontaine, G., Brassard, P., Green, E., et al. 2008, A\&A, 486, L39

Green, E. M., Fontaine, G., Reed, M. D., et al. 2003, ApJ, 583, L31

Iglesias, C. A., \& Rogers, F. J. 1996, ApJ, 464, 943

Jeffery, C. S., \& Saio, H. 2006a, MNRAS, 371, 659

Jeffery, C. S., \& Saio, H. 2006b, MNRAS, 372, L48

Jeffery, C. S., \& Saio, H. 2007, MNRAS, 378, 379

Kilkenny, D., Koen, C., O’Donoghue, D., \& Stobie, R. S. 1997, MNRAS, 285, 640

Van Grootel, V., Charpinet, S., Fontaine, G., \& Brassard, P. 2008, A\&A, 483, 875

Woudt, P. A., Kilkenny, D., Zietsman, E., et al. 2006, MNRAS, 371, 1497 